\title{
SOME p-GROUPS OF WEILANDT LENGTH THREE
}

\author{
Elizabeth A. OrMERod
}

\begin{abstract}
Finite $p$-groups of Wielandt length 1 are groups in which every subgroup is normal and are Dedekind groups. When the prime is odd therefore a finite $p$-group of Wielandt length 1 is Abelian. For an odd prime, a finite p-group of Wielandt length 2 has nilpotency class at most 3 and for such a goup to have class 3 there must be a 2-generator subgroup of this class. In this paper it is shown that for any prime $p>3$ a finite $p$-group of Wielandt length 3 has nilpotency class at most 4, and for such a group to have class 4 there must be a 2-generator subgroup with this class. Two families of $p$-groups of Wielandt length 3 are described. One is a family of 3-generator groups with the property that each group modulo its Wielandt subgroup has class 2 , the other is a family of 2-generator groups with the property that each group modulo its Wielandt subgroup has class 3 .
\end{abstract}

\section{INTRODUCTION}

This paper is a contribution to the study of groups of Wielandt length three. The Wielandt subgroup is the intersection of the normalisers of all the subnormal subgroups of a group and was initially studied by Wielandt [8]. He showed among other things that for a finite group the Wielandt subgroup is a non trivial characteristic subgroup of the group. This allows an ascending normal series to be defined for a group $G$. We let $\omega(G)$ be the Wielandt subgroup of $G$ and define $\omega_{0}(G)=1$, and then if $\omega_{n}(G)$ has been defined, we set $\omega_{n+1}(G) / \omega_{n}(G)=\omega\left(G / \omega_{n}(G)\right)$. For a finite group $G, \omega_{n}(G)=G$ for some integer $n$. The smallest such $n$ is called the Wielandt length of $G$. The class of groups having Wielandt length at most $n$ is denoted by $\mathcal{W}_{n}$.

The groups considered in this paper will all be finite $p$-groups and for these the Wielandt subgroup becomes the intersection of the normalisers of all subgroups. Finite $p$-groups in $\mathcal{W}_{1}$ are groups in which every subgroup is normal and these groups are well known as Dedekind groups.

The structure of finite $p$-groups in $\mathcal{W}_{2}$ for an odd prime $p$ has been described in Ormerod [6]. These groups have class at most 3 and only attain this class if there is 2-generator subgroup having class 3 . Thus 2-generator groups play an important role in the description of groups of odd order in $\mathcal{W}_{2}$, and for each odd prime an infinite

Received 23rd December, 1997

Copyright Clearance Centre, Inc. Serial-fee code: 0004-9729/98 \$A2.00+0.00. 
family of 2-generator groups is defined of class 3 and in $\mathcal{W}_{2}$. Any $p$-group $(p \geqslant 3)$ in $\mathcal{W}_{2}$ is the homomorphic image of the second nilpotent product of one of these groups with a group of class 2 . This description has been used by Ali [1] in his Ph.D thesis to describe supersoluble groups of Wielandt length 2 whose order is co-prime to 6 .

A description of finite 2-groups in $\mathcal{W}_{2}$ has not yet been completed. Mahdavi [4] in his classification of 2-generator 2-groups with many groups subnormal includes a description of the 2-generator 2-groups in $\mathcal{W}_{2}$. In this description there are three infinite families and one group of order 16. This indicates that the classification of all finite 2-groups in $\mathcal{W}_{2}$ will be quite complex. It is further complicated by the fact that 2-groups in $\mathcal{W}_{2}$ with three or more generators when factored by the Wielandt subgroup may be Hamiltonian rather than just Abelian.

This complexity of classification of 2-groups in $\mathcal{W}_{2}$ results from the fact that having class 2 or 3 , these groups need not be regular. Indeed 3-groups of class 3 need not be regular, and this is reflected in the slightly more complex situation for 3-groups in the description in [6], although this is most significant when the exponent of the group is small.

In considering $p$-groups in $\mathcal{W}_{3}$ we have chosen to consider groups when the prime is greater than 3 . Since $p$-groups in $\mathcal{W}_{2}$ have class at most 3 , and since the Wielandt subgroup is in the second centre of a group, $p$-groups in $\mathcal{W}_{3}$ can have class at most 5 . The first result of the paper is that $p$-groups $(p>3)$ in $\mathcal{W}_{3}$ have class at most 4 . This means that the groups we consider are all regular. Again we show the importance of 2-generator groups in $\mathcal{W}_{3}$ by proving the following theorem.

Theorem 3.9. Let $G$ be a $p$-group, where $p>3$. If $G \in \mathcal{W}_{3}$ and every 2-generator group has class at most 3 , then $G$ has class at most 3 .

Finally, when considering $p$-groups in $\mathcal{W}_{3}$ two different situations may arise. For a group $G \in \mathcal{W}_{3}$ it is possible that $G / \omega(G)$ has class 2 or 3 , and these cases are considered separately. When $G / \omega(G)$ has class 2 we are able to describe an infinite family of 3-generator groups for each prime with the property that any 3-generator group in $\mathcal{W}_{3}$ must be a homomorphic image of one of these groups. When $G / \omega(G)$ has class 3 we describe an infinite family of 2 -generator groups with the same property for 2-generator groups.

\section{THE NILPOTENCY CLASS}

This section provides a proof that a $p$-group in $\mathcal{W}_{3}(p>3)$ has nilpotency class at most four. It begins with a preliminary lemma, which relies on a result of Schenkman [7] that the norm of a group is between the centre and second centre of a group. For a nilpotent group, the norm and the Wielandt subgroup coincide. 
LEMMA 2.1. Let $G$ be a finite nilpotent group and let $w$ be an element in $\omega_{2}(G)$. Then for any $x$ and $y$ in $G,[w, x, x, y]=1$.

Proof: Since $w \in \omega_{2}(G),[w, x]=x^{n} \bmod \omega(G)$ for some integer $n$ which depends on $x$ and $w$. This means that $x^{-n}[w, x]$ is in $\omega(G)$ and is in the second centre of $G$. Hence $1=\left[x^{-n}[w, x], x, y\right]=[w, x, x, y]$ for any $x$ and $y$ in $G$.

The main result relies heavily on the description of $p$-groups in $\mathcal{W}_{2}$ given in [6]. We assume that $p$ is a prime and we define the following groups.

$$
H(p, r)=\left\langle x, y:[y, x, x]=x^{p^{r}},[y, x, y]=y^{p^{r}}, x^{p^{2 r}}=y^{p^{2 r}}=[x, y]^{p^{r}}=1\right\rangle
$$

where $p>3$ and $r$ is a positive integer. These groups have class 3 and were initially introduced by Hobby [3] when investigating groups in which the normaliser of every subgroup is normal. Also let $L_{n}\left(p^{r}\right)$ be the free group of rank $n$ in the variety of groups that are nilpotent of class at most 2 , and have exponent $p^{r}$. To complete this description of groups in $\mathcal{W}_{2}$ we need to define the $k$ th nilpotent product of two groups $A$ and $B$. Let $F$ be the free product of $A$ and $B$, let $k$ be any natural number and let $\gamma_{k}(F)$ be the $k$ th term of the lower central series of $F$. The $k$ th nilpotent product of $A$ and $B$ is the factor group $F /\left(\gamma_{k}(F) \cap[A, B]\right)$ and is denoted by $A * \mathcal{N}_{k} B$. $[6$, Theorem $A]$ includes the following result.

Theorem 2.2. Let $p$ be a prime, $p>3$, and let $G$ be a finite $p$-group. Then $G$ has Wielandt length two if and only if $G$ is a homorphic image of

$$
H(p, r) * \mathcal{N}_{2} L_{n}\left(p^{r}\right)
$$

where $r$ and $n$ are positive integers.

THEOREM 2.3. Let $G$ be $p$-group of Wielandt length 3 . If $p>3$ then $G$ has nilpotency class at most 4.

Proof: Since $G$ has Wielandt length $3, G / \omega(G)$ must be isomorphic to a quotient of $H(p, r) * \mathcal{N}_{2} L_{t-2}\left(p^{r}\right)$ where $t$ and $r$ are positive integers and $t \geqslant 2$. Also $G / \omega_{2}(G)$ is Abelian. Hence there are generators $g_{1}, \ldots, g_{n}$ for $G$ such that

$$
\begin{aligned}
\omega(G) \supseteq\left\{g_{1}^{p^{2 r}}, g_{2}^{p^{2 r}}, g_{1}^{-p^{r}}\left[g_{2}, g_{1}, g_{1}\right], g_{2}^{-p^{r}}\left[g_{2}, g_{1}, g_{2}\right], g_{3}^{p^{r}}, \ldots, g_{t}^{p^{r}},\right. \\
\left.\left.\qquad g_{i}, g_{j}, g_{k}\right] \text { for }\{i, j, k\} \neq\{1,2\}, g_{t+1}, \ldots, g_{n}, \text { where } 2 \leqslant t \leqslant n\right\} .
\end{aligned}
$$

Since $G / \omega(G)$ has class at most three, $G$ can have class at most five. We aim to show that all commutators in the generators of weight five are trivial.

Using the inclusion $\omega(G) \subseteq \zeta_{2}(G)$ we note that $\left[g_{i}, g_{j}, g_{k}, g_{r}, g_{s}\right]=1$ for $\{i, j, k\} \neq$ $\{1,2\}$. Further since $G / \omega_{2}(G)$ is Abelian, $\left[g_{2}, g_{1}\right] \in \omega_{2}(G)$. From 2.1 this means that

$$
\left[g_{2}, g_{1}, x, x, y\right]=1 \quad \text { for all } \quad x, y \in G
$$


Hence, $\left[g_{2}, g_{1}, g_{1}, g_{1}, g_{k}\right]=1$ and $\left[g_{2}, g_{1}, g_{2}, g_{2}, g_{k}\right]=1$, for $1 \leqslant k \leqslant n$. By substituting $x_{1} x_{2}$ for $x$ in 2.4, and expanding, we further deduce that

$$
\left[g_{2}, g_{1}, x_{1}, x_{2}, y\right]\left[g_{2}, g_{1}, x_{2}, x_{1}, y\right]=1 \quad \text { for all } \quad x_{1}, x_{2}, y \in G
$$

From this it is clear that $\left[g_{2}, g_{1}, g_{1}, g_{j}, g_{k}\right]\left[g_{2}, g_{1}, g_{j}, g_{1}, g_{k}\right]=1$, for $1 \leqslant j, k \leqslant n$. However, if we require that $j \geqslant 3$, then $\left[g_{2}, g_{1}, g_{j}, g_{1}, g_{k}\right]=1$ and so $\left[g_{2}, g_{1}, g_{1}, g_{j}, g_{k}\right]=$ 1 , for $3 \leqslant j \leqslant n$ and $1 \leqslant k \leqslant n$. Similarly, we can also show that $\left[g_{2}, g_{1}, g_{2}, g_{j}, g_{k}\right]=1$ for $3 \leqslant j \leqslant n$ and $1 \leqslant k \leqslant n$.

The only commutators in the generators that have not been shown to be trivial are those of the form $\left[g_{2}, g_{1}, g_{1}, g_{2}, g_{k}\right]$ and $\left[g_{2}, g_{1}, g_{2}, g_{1}, g_{k}\right]$, for $1 \leqslant k \leqslant n$. From 2.5 , it is clear that

But

where

Hence

and so

$$
\begin{aligned}
{\left[g_{2}, g_{1}, g_{1}, g_{2}, g_{k}\right]\left[g_{2}, g_{1}, g_{2}, g_{1}, g_{k}\right] } & =1 \quad \text { for } \quad 1 \leqslant k \leqslant n . \\
{\left[g_{2}, g_{1}, g_{1}, g_{2}\right] } & =\left[g_{2}, g_{1}, g_{2}, g_{1}\right] w \\
w & \in\left[G^{\prime}, G^{\prime}\right] \cap\left\langle g_{1}, g_{2}\right\rangle . \\
w & \in \gamma_{5}(G),
\end{aligned}
$$$$
\left[g_{2}, g_{1}, g_{1}, g_{2}, g_{k}\right]=\left[g_{2}, g_{1}, g_{2}, g_{1}, g_{k}\right] \text {. }
$$

Since $p \neq 2$, from 2.6, we deduce that $\left[g_{2}, g_{1}, g_{1}, g_{2}, g_{k}\right]=\left[g_{2}, g_{1}, g_{2}, g_{1}, g_{k}\right]=1$ for $k \in\{1, \ldots, n\}$. Since all commutators of weight 5 in the generators are trivial, we conclude that $G$ has class at most 4 .

\section{TWO GENERATOR GROUPS}

In the description of $p$-groups in $\mathcal{W}_{2}$ it is clear that the 2-generator subgroups play an important role, and if a group in $\mathcal{W}_{2}$ achieves the maximum class, then a 2-generator subgroup must achieve this class. For groups in $\mathcal{W}_{3}$ we have a similar situation and in this section we show that if $G$ is a $p$-group in $\mathcal{W}_{3}$ where $p>3$, and if every 2generator subgroup has class 3 , then $G$ has class 3 . From Theorem 2.3 the groups we are considering have class at most 4 . This together with the assumption that $p>3$ ensures the groups are regular. An important property of a regular group is highlighted in the next lemma and its corollary. This property is used extensively in this section and throughout the paper.

LEMma 3.1. (See Cooper [2, 5.3]). If $G$ is a finite group whose Sylow subgroups are regular, every power automorphism is universal.

Elements of the Wielandt subgroup induce power automorphisms on group elements, so the corollary follows immediately. 
COROLlary 3.2. Let $G$ be a regular p-group with $w \in \omega(G)$. Then there is an integer $n$ such that $[w, g]=g^{n}$ for all $g \in G$.

In the following series of lemmas we make the assumption that $G$ is a $p$-group, $p>3, G \in \mathcal{W}_{3}$ and every 2-generator subgroup has class at most 3 .

LEMMA 3.3. Let $a$ and $b$ be elements of $G$ such that $[b, a, a]=a^{\beta p^{r}} \bmod \omega(G)$, where $\beta$ and $r$ are integers and $(\beta, p)=1$. Then

(i) $\left[a^{\beta p^{r}}, x, y\right]=1$ for all $x$ and $y$ in $G$,

(ii) $\left[a^{p^{r}}, b, a\right]=\left[a^{p^{r}}, b, b\right]=1$, and

(iii) $\left[a^{\beta p^{r}}, b\right]=1$ if and only if $\left[a^{p^{r}}, b\right]=1$.

These calculations are straightforward commutator calculations which rely on the assumption that 2-generator subgroups of $G$ have class at most 3, and the fact that the Wielandt subgroup is in the second centre of $G$.

Lemma 3.4. Let $a \in G$ be an element of maximal order in $G$. Then for any $b \in G,[b, a, a] \in \zeta(G) \subseteq \omega(G)$.

Proof: For each $b \in G,[b, a] \in \omega_{2}(G)$. Hence

$$
[b, a, a]=a^{\beta_{b} \mathrm{p}^{r} b} \bmod \omega(G)
$$

for integers $\beta_{b}, r_{b}$ where $\left(\beta_{b}, p\right)=1$. Since $G$ is regular there are integers $\nu$ and $n$ depending on $a$ and $b$ only such that

$$
\left[a^{-\beta_{b} p^{p_{b}}}[b, a, a], g\right]=g^{\nu p^{n}} \quad \text { for all } g \in G .
$$

We use the assumption that 2-generator subgroups have class at most 3 to get:

$$
\left[a^{-\beta_{b} p^{r_{b}}}[b, a, a], a\right]=a^{\nu p^{n}}=[b, a, a, a]=1 .
$$

Since $a$ has maximal order in $G$, we conclude that $g^{\nu p^{n}}=1$ for all $g \in G$. From here we note that

$$
\left[a^{-\beta_{b} p^{r_{b}}}[b, a, a], b\right]=\left[a^{-\beta_{b} p^{r_{b}}}, b\right][b, a, a, b]=1,
$$

using the result that $G$ has class at most 4 . Further, $[b, a, a, b]=1$ and so $\left[a^{-\beta_{b} p^{r} b}, b\right]=$ 1. This implies that $\left[a^{\beta_{b} p^{r_{b}}}, b\right]=1$ and by 3.1 (iii) also $\left[a^{p^{r_{b}}}, b\right]=1$.

Let $c$ be any element of $G$. Then there are integers $\beta_{c}$ and $r_{c}$ such that

$$
[c, a, a]=a^{\beta_{c} p^{r_{c}}} \bmod \omega(G),
$$

where $\left(\beta_{c}, p\right)=1$, and $\left[a^{p^{r c}}, c\right]=1$. Also

$$
1=\left[a^{-\beta_{b} p^{\tau_{b}}}[b, a, a], c\right]=\left[a^{-\beta_{b} p^{\tau_{b}}}, c\right][b, a, a, c]
$$


and from this we deduce that

$$
[b, a, a, c]=\left[c, a^{-\beta_{b} p^{r_{b}}}\right]=\left[a^{\beta_{b} p^{r_{b}}}, c\right] .
$$

In a similar way

$$
[c, a, a, b]=\left[b, a^{-\beta_{c} p^{r_{c}}}\right]=\left[a^{\beta_{c} p^{r_{c}}}, b\right] .
$$

Now suppose that $[b, a, a, c] \neq 1$. Then $\left[a^{\beta_{c} p^{r_{c}}}, b\right] \neq 1$ and, from 3.1(iii), $\left[a^{p^{r_{c}}}, b\right] \neq 1$. This means that $r_{b}<r_{c}$, which in turn means that

$$
1=\left[a^{p^{r_{c}}}, b\right]=\left[a^{\beta_{c} p^{r_{c}}}, b\right]=[c, a, a, b]
$$

Since 2-generator subgroups have class at most three, and $G$ has class at most four,

$$
1=[b c, a, a, b c]=[b, a, a, b][b, a, a, c][c, a, a, b][c, a, a, c]=[b, a, a, c] .
$$

From this we conclude that $[b, a, a]$ is central is $G$.

LEMMA 3.5. If $a$ is of maximal order in $G$, and $b$ is any element of $G$ such that $|b|<|a|$, then $|a b|=|a|$.

PROOF: Let $|a|=p^{m}$ for some integer $m$. Then

$$
(a b)^{p^{m-1}}=a^{p^{m-1}} b^{p^{m-1}}[a, b]^{c_{1}}[a, b, b]^{c_{2}}[a, b, a]^{c_{3}}
$$

where $p^{m-1} \mid c_{i}, i=1,2,3$, since $G$ is regular. Again using the regularity of $G$ and the assumption that $|b|<p^{m}$, we conclude that $[a, b]^{c_{1}},[a, b, b]^{c_{2}}$ and $[a, b, a]^{c_{3}}$ are all trivial. Hence $(a b)^{p^{m-1}}=a^{p^{m-1}} \neq 1$, while clearly $(a b)^{p^{m}}=1$. This gives the required result.

LEMma 3.6. Let $a$ be of maximal order in $G$, then $[b, a, b] \in \zeta(G)$ for any $b \in G$.

PROOF: If $|b|=|a|$ then by $3.4[b, a, b] \in \zeta(G)$. So assume $|b|<|a|$, and hence $|a b|=|a|$. Then by 3.4 and $3.5[b, a b, a b] \in \zeta(G)$. But $[b, a b, a b]=[b, a, a][b, a, b]$, using the assumption on 2-generator groups, and the result that $[b, a, a] \in \zeta(G)$. Hence $[b, a, b]$ is central in $G$.

LEMMA 3.7. If $b$ and $c$ are any elements of $G$, then $[b, c, c] \in \zeta(G)$.

Proof: Let $a$ be of maximal order in $G$. Then by $3.6[b c, a, b c] \in \zeta(G)$. Also

$$
[b c, a, b c]=[b, a, b][b, a, c][c, a, b][c, a, c] \bmod \zeta(G)
$$

and so

$$
[b, a, c][c, a, b] \in \zeta(G)
$$


If $|c|<|a|$, then $|a c|=|a|$, and by $3.8,[b, a c, c][c, a c, b] \in \zeta(G)$. But

$$
[b, a c, c][c, a c, b]=[b, a, c][b, c, c][c, a, b] \bmod \zeta(G) .
$$

This, together with 3.8 , implies that $[b, c, c] \in \zeta(G)$. Clearly, if $|c|=|a|$ then from 3.4 $[b, c, c] \in \zeta(G)$, giving the required result.

Theorem 3.9. Let $G$ be a $p$-group, where $p>3$. If $G \in \mathcal{W}_{3}$ and every 2-generator group has class at most 3 , then $G$ has class at most 3 .

Proof: Let $a, b$ and $c$ be any elements of $G$. From 3.7, $[b, a c, a c] \in \zeta(G)$ and

$$
[b, a c, a c]=[b, a, a][b, a, c][b, c, a][b, c, c] \bmod \zeta(G) .
$$

Hence $[b, a, c][b, c, a] \in \zeta(G)$, and

$$
[b, a, c]=[c, b, a] \bmod \zeta(G) .
$$

If $a$ is of maximal order we can use 3.6 to get

$$
[b, a, c]=[a, c, b] \bmod \zeta(G) .
$$

Using the Jacobi-Witt identity and the assumption that $G$ has class at most 4 , we get

$$
[b, a, c][a, c, b][c, b, a] \in \zeta(G) .
$$

With the equations above, this implies that

$$
[b, a, c]^{3} \in \zeta(G)
$$

and since $(p, 3)=1$, we conclude that $[b, a, c] \in \zeta(G)$.

If $a$ is not of maximal order let $d$ be an element of maximal order in $G$. Then $[b, a d, c] \in \zeta(G)$ and

$$
[b, a d, c]=[b, a, c][b, d, c] \bmod \zeta(G) .
$$

From this it is clear that $[b, a, c] \in \zeta(G)$ and this is true for any $a, b, c$ in $G$. Hence $G$ has class at most 3 .

\section{GRoups of WielandT LENGTH Three. (I)}

In this section we look at the structure of some groups whose factor groups via the Wielandt subgroup have class 2. These groups have maximum class 4 and it is the groups that attain this maximum in which we are interested. We consider 3-generator groups, although the previous section has shown the importance of 2-generator groups. 
In this situation it is just as easy to work with the 3-generator groups, and the results give a clearer picture as they include non-metabelian groups, as well as metabelian groups. The 2-generator are all metabelian since they have nilpotency class at most 4 . We assume that $p>3$ and hence the groups are regular.

Let us assume that $G$ is a 3-generator $p$-group with generators $a, b$ and $c$. Without any loss of generality we can assume that $|a| \geqslant|b| \geqslant|c|$ and using the regularity of $G$ we can also assume that $\langle a\rangle \cap\langle b\rangle=\langle a\rangle \cap\langle c\rangle=1$ (see Ormerod [6, 4.1]). Since 2-generator groups of class 4 are metabelian and since any weight three commutator is in the Wielandt subgroup, this immediately leads to some easy conclusions: namely $[b, a, a, b]=[b, a, b, a] \in\langle a\rangle \cap\langle b\rangle$ which means that $[b, a, a, b]=1$. Similiarly, $[c, a, c, a]=$ 1 .

As noted earlier (3.1 and 3.2) since $G$ is a regular p-group and power automorphisms of regular groups are universal, we can also assume that if $w \in \omega(G)$ then there is an integer $n$ such that $[w, g]=g^{n}$ for all $g \in G$. This allows us to make the further conclusions that since $[b, a, b, a]=1$ and $|a| \geqslant|b| \geqslant|c|$ then $[b, a, b, b]=1$ and $[b, a, b, c]=1$. Similarly we can also conclude that $[b, a, a, c]=1$ and $[c, a, c, b]=[c, a, c, c]=1$.

LEMMA 4.1. Let $G$ be a p-group of Wielandt length 3. Then the following law holds in $G$.

$$
[x, y, z, t]=[x, y, t, z][[x, y],[z, t]]
$$

Proof: It is well known that in any group

$$
[x, y, z t]=[x, y, t][x, y, z]^{t}=[x, y, t][x, y, z][x, y, z, t]
$$

There is a similar expression for $[x, y, t z]$ and since $G$ has class at most four and hence all these commutators commute we can deduce that

$$
[x, y, z, t][x, y, t, z]^{-1}=[x, y, z t][x, y, t z]^{-1}
$$

Now $z t=t z[z, t]$ and we can calculate that

$$
[x, y, z t][x, y, t z]^{-1}=[[x, y],[z, t]]
$$

This is the required result.

We use this result and the previous calculations to derive that $[b, a, c, a]=$ $[[b, a],[c, a]]$ and $[c, a, b, a]=[c, a, a, b][[c, a],[b, a]]$. Together these give: $[c, a, b, a]$ $[b, a, c, a]=[c, a, a, b]$. As above this implies that $[c, a, a, b]=1$ and $[c, a, b, a][b, a, c, a]=$ 1. Since $G$ has class at most four we can derive from the Jacobi-Witt identity that 
$[c, a, b, a][a, b, c, a][b, c, a, a]=1$. Hence once one of these commutators is determined, it fixes the value of the other two.

Using similar techniques we also conclude that $[c, b, c, a]=[c, b, a, c][c, a, b, c]=1$ and from that $[c, b, c, b]=[c, b, c, c]=1$ and that $[c, b, b, a]=[c, b, a, b][a, b, c, b]=1$ and from this that $[c, b, b, b]=[c, b, b, c]=1$.

The only commutators that remain non-trivial at this stage are

$$
\begin{array}{lll}
{[b, a, a, a]} & {[c, a, a, a]} & \\
{[c, a, b, a]} & {[b, a, c, a]} & {[c, b, a, a]} \\
{[c, a, b, b]} & {[b, a, c, b]} & {[c, b, a, b]} \\
{[c, a, b, c]} & {[b, a, c, c]} & {[c, b, a, c]}
\end{array}
$$

where as we noted before in the lines containing three commutators once the value of one of them is determined the value of the other two is fixed.

Let us assume that $[b, a, c, a]=a^{m p^{\alpha}}$ where $(m, p)=1$. Since $G$ is regular this implies that $[b, a, c, b]=b^{m p^{\alpha}}$ and $[b, a, c, c]=c^{m p^{\alpha}}$. The relationships already discussed then allow us to say that $[c, a, b, a]=a^{-m p^{\alpha}}$ and similarly, $[c, a, b, b]=b^{-m p^{\alpha}}$, $[c, a, b, c]=c^{-m p^{\alpha}}$. We have further established that $[c, b, a, b]=[b, a, c, b]$ and using the (extended) Jacobi-Witt identity we have

$$
[b, a, c, b][a, c, b, b][c, b, a, b]=1 \text {. }
$$

Using the assigned values this implies that $b^{3 m p^{\alpha}}=1$. Since $(3 m, p)=1$ this is sufficient to conclude that $b^{p^{\alpha}}=1$. In a similar way, since $[c, b, a, c]=[a, c, b, c]$ we can also conclude that $c^{p^{\alpha}}=1$. Let us summarise the situation at this stage.

LeMMA 4.2. Let $G$ be a 3-generator p-group in $\mathcal{W}_{3}$ with the property that $G / \omega(G)$ has class 2. If generators of $G$ are $a, b$ and $c$, with $|a| \geqslant|b| \geqslant|c|$, and $\langle a\rangle \cap\langle b\rangle=\langle a\rangle \cap\langle c\rangle=1$, then

(i) $\gamma_{4}(G)$ is cyclic, and is a subgroup of $\langle a\rangle$,

(ii) the only nontrivial weight four commutators in the generators are from the following list:

$$
[b, a, c, a],[c, a, b, a],[c, b, a, a],[b, a, a, a], \text { and }[c, a, a, a]
$$

Let us assume that $|[c, a, a, a]| \leqslant|[b, a, a, a]|$ and that $[c, a, a, a]=a^{n p^{\gamma}}$ and $[b, a, a, a]=a^{t p^{\beta}}$ where $(n, p)=(t, p)=1$ and $\gamma \geqslant \beta, \gamma$ and $\beta$ both positive integers. Put $c^{\prime}=c^{t} b^{-n p^{\gamma-\beta}}$. Then $G=\left\langle a, b, c^{\prime}\right\rangle,\left[c^{\prime}, a, a, a\right]=1$, all trivial commutators remain trivial if $c$ is replaced by $c^{\prime}$ and the nontrivial commutators maintain the same 
relationship with each other if $c$ is replaced by $c^{\prime}$. Hence we assume that $[c, a, a, a]=1$ and the nontrivial commutators are

$$
[b, a, c, a],[c, a, b, a],[c, b, a, a] \text {, and }[b, a, a, a] .
$$

Let us now assume that $[b, a, a, a]=a^{p^{\beta}}$ and that $[b, a, c, a]=a^{p^{\gamma}}$. It may be necessary first to choose an appropriate power of $b$ and then an appropriate power of $c$ to ensure this simplicity of representation. This also means that $[c, a, b, a]=a^{-p^{\gamma}}$ and $[b, c, a, a]=$ $a^{2 p^{\gamma}}$.

If $|[b, a, a, a]| \leqslant|[c, a, a, a]|$ a similar argument can be used with the roles of $b$ and $c$ reversed to get a similar list of non trivial commutators. Although we have assumed that $|b| \geqslant|c|$ this was not used in the preceding argument, and as the following theorem shows it is possible for $b$ and $c$ to have the same order. The following result summarises this argument and provides further information.

ThEOREM 4.3. Let $G$ be a 3-generator p-group. Then $G$ has Wielandt length 3 and $G / \omega(G)$ has nilpotency class 2 if and only if $G$ is isomorphic to a homomorphic image of one of the following groups:

$$
\begin{aligned}
G(\beta, \gamma)=\left\langle a, b, c: a^{p^{p^{\beta}}}=b^{p^{\beta}}=c^{p^{\beta}}=1,\left[a^{p^{\beta}}, b\right]=\left[a^{p^{\beta}}, c\right]=1,\right. \\
{[b, a, a, a]=a^{p^{\beta}},[b, a, c, a]=a^{p^{\gamma}},[c, a, b, a]=a^{-p^{\gamma}},[b, c, a, a]=a^{2 p^{\gamma}}, } \\
{[b, a, b, a]=[b, a, b, b]=[b, a, b, c]=[b, a, a, c]=[c, a, a, b]=} \\
{[c, a, a, a]=[c, a, c, a]=[c, a, c, b]=[c, a, c, c]=[c, b, b, a]=} \\
{[c, b, b, b]=[c, b, c, a]=[c, b, c, b]=[c, b, c, c]=[b, a, c, b]=} \\
{[b, a, c, c]=1\rangle, \quad \text { where } \beta \leqslant \gamma \leqslant 2 \beta . }
\end{aligned}
$$

Proof: From what has already been said we have essentially derived this presentation for a group $G$ satisfying the given conditions, except for the restrictions on $\beta$, and the orders of the generators. If $\gamma \geqslant \beta$, it easily follows from the preceding work, that the maximum order of the generators is as given in the statement of the theorem. We need to consider the case $\beta>\gamma$. In fact we assume that $\beta \geqslant \gamma$ and show that each of the groups with $\beta \geqslant \gamma$ is isomorphic to the group $G(\gamma, \gamma)$ and so is included by looking at the case $\beta=\gamma$.

Put $a^{\prime}=a c^{m p^{\beta-\gamma}}$, for some integer $m$. Then

$$
\begin{aligned}
{\left[b, a^{\prime}, a^{\prime}, a^{\prime}\right] } & =[b, a, a, a]\left[b, a, c^{n}, a\right]\left[b, c^{n}, a, a\right] \quad \text { where } n=m p^{\beta-\gamma} \\
& =a^{p^{\beta}} a^{m p^{\beta}} a^{2 m p^{\beta}} .
\end{aligned}
$$

If we choose $m$ so that $3 m+1 \equiv p^{2 \gamma-\beta} \bmod p^{2 \gamma}$, then $\left[b, a^{\prime}, a^{\prime}, a^{\prime}\right]=1$ and $\left[b, a^{\prime}, c, a^{\prime}\right]=$ $a^{p^{\gamma}}$, and all other relations hold with $a^{\prime}$ replacing $a$. 
We now show that the groups $G(\beta, \gamma)$ have Wielandt length 3 . We show that $\gamma_{3}(G(\beta, \gamma)) \subseteq \omega(G(\beta, \gamma))$, and this implies that $G / \omega(G)$ has niplotency class at most 2. It is clear that $[b, a, b],[c, a, a],[c, a, c],[c, b, b]$ and $[c, b, c]$ are central in $G$ and hence belong in $\omega(G)$. Let $g$ be any element of $G$. We can write $g=a^{r} b^{s} c^{t} u$ where $u \in \gamma_{2}(G)$. Then

$$
\begin{aligned}
{[[b, a, a], g] } & =\left[b, a, a, a^{r}\right]\left[b, a, a, b^{s}\right]\left[b, a, a, c^{t}\right] \\
& =[b, a, a, a]^{r} \\
& =a^{r p^{\beta}} \\
& =g^{p^{\beta}} \quad \text { by the regularity of } G .
\end{aligned}
$$

Hence $[b, a, a] \in \omega(G)$ and in a similar way $[b, a, c],[c, a, b]$ and $[c, b, a]$ all belong in $\omega(G)$. Since $G$ has class four, we deduce that $G / \omega(G)$ does have class 2, and therefore Wielandt length 2. Hence $G$ has Wielandt length 3 .

It is worthwhile at this stage to make a few comments about this family of groups $G(\beta, \gamma)$, as a mere presentation of a group can raise more questions than it answers.

1. $G(\beta, \gamma)$ is not metabelian, unless $\gamma=2 \beta$. From Lemma 4.1 we have that $[b, a, c, a]=[b, a, a, c][[b, a],[c, a]]$, so from the relations of the group $[b, a, c, a]=[[b, a],[c, a]]$ which is nontrivial, unless $\gamma=2 \beta$.

2. All commutators have order at most $p^{\beta}$, since $a^{p^{\beta}}$ is central, $b^{p^{\beta}}=c^{p^{\beta}}=$ 1 , and $G(\beta, \gamma)$ is regular.

3. If we put $N=\left\langle a^{p^{\beta}}\right\rangle$ then $N$ is central in $G(\beta, \gamma)$ and $G(\beta, \gamma) / N \cong$ $F_{3}\left(\mathcal{N}_{3} \wedge \mathcal{B}_{p^{\beta}}\right)$, the free group of rank 3 in the variety of all groups that are nilpotent of class at most 3 , and have exponent dividing $p^{\beta}$. This follows from the previous comment, and the lack of any other relations on the group. It also relies on the group being regular.

4. $|G(\beta, \gamma)|=p^{15 \beta}$. This follows from the following calculations: $\left|\gamma_{4}(G)\right|=$ $p^{\beta},\left|\gamma_{3}(G) / \gamma_{4}(G)\right|=p^{8 \beta},\left|\gamma_{2}(G) / \gamma_{3}(G)\right|=p^{3 \beta}$, and $\left|g / \gamma_{2}(G)\right|=p^{3 \beta}$, where these numbers come from the number of basic commutators of each weight.

5. None of these comments so far make any reference to $\gamma$ and this raises the question whether $\gamma$ makes any difference. However, if we look at the upper central series of each of these groups the influence of $\gamma$ becomes apparent. Firstly we look at the centre of $G(\beta, \gamma)$ :

$$
\begin{array}{r}
\zeta(G(\beta, \gamma))=\left\langle a^{p^{\beta}},[b, a, b],[c, a, a],[c, a, c],[c, b, b],[c, b, c],\right. \\
\left.[b, a, c][b, a, a]^{-p^{\gamma-\beta}},[c, a, b][b, a, a]^{p^{\gamma-\beta}}\right\rangle .
\end{array}
$$


It is clear that the elements listed as generators are central. It takes a small calculation to show that nothing else can belong to the centre. The order of the centre is $p^{8 \beta}$.

Looking at the second centre is more difficult, but is it helpful in showing that these groups are different. For $\gamma<3 \beta / 2$ we have:

$$
\zeta_{2}(G(\beta, \gamma))=\left\langle[b, a, a],[c, a]^{p^{2 \beta-\gamma}},[c, b]^{p^{2 \beta-\gamma}}, \zeta(G(\beta, \gamma))\right\rangle
$$

and $\left|\zeta_{2}(G(\beta, \gamma))\right|=p^{7 \beta+2 \gamma}$.

For $\gamma \geqslant 3 \beta / 2$ we have:

$$
\zeta_{2}(G(\beta, \gamma))=\left\langle[b, a, a],[c, a]^{p^{2 \beta-\gamma}},[c, b][b, a]^{2 p^{\gamma-\beta}}, \zeta(G(\beta, \gamma))\right\rangle
$$

and $\left|\zeta_{2}(G(\beta, \gamma))\right|=p^{9 \beta+\gamma}$.

Again it is easy to see in each case that the list of generators belongs in the second centre. It takes a little more work to show that the second centre can not contain any other generators.

Before leaving this section it seems useful to give some indication of where this result about 3-generator groups may lead. It would be nice to know what any $p$-group $G$ that has the property that $G / \omega(G)$ has nilpotency class 2 looks like. Moving into the situation of four generators, let us assume that we have a goup $G$ with generators $a, b$, $c$ and $d$. Again we can assume that $|a| \geqslant|b| \geqslant|c| \geqslant|d|$ and that $\langle a\rangle \cap\langle b\rangle=\langle a\rangle \cap\langle c\rangle=$ $\langle a\rangle \cap\langle d\rangle=1$. Using the Lemma, 4.2, we can make conclusions about $\langle a, b, c\rangle,\langle a, b, d\rangle$ and $\langle a, c, d\rangle$. Using the information gained from these subgroups, and the regularity of the group we can make further conclusions about $\langle b, c, d\rangle$. We find that the only possible nontrivial commutators of weight in any three (or fewer) of the generators are those with an $a$ in the fourth position, and since $\gamma_{3}(G)$ is in the Wielandt subgroup, these commutators are all in $\langle a\rangle$. The next, perhaps surprising, result is that using this information, and Lemma 4.1, all the commutators in the four generators are trivial. It would seem that $G / \gamma_{4}(G)$ is isomorphic to the free group of rank four in the variety of all groups that are nilpotent of class at most 3 , and have exponent dividing $p^{\beta}$ for some integer $\beta$.

For groups with more generators it seems clear that the same pattern will continue and it is possibly true that if $G$ is a finite $p$-group of Wielandt length 3 and nilpotency class four, with the property that $G / \omega(G)$ has nilpotency class 2 , then $\gamma_{4}(G)$ is cyclic. Some of the questions raised are:

If a $p$-group has Wielandt length four, and its factor group via the Wielandt subgroup is like the groups just described, is it possible for the group to have class six?

Under what conditions do a $p$-group and its factor group via the Wielandt subgroup differ in nilpotency class by two? 


\section{Groups of Wielandt length three. (II)}

In the previous section we considered $p$-groups in $\mathcal{W}_{3}$ whose factor groups via the Wielandt subgroup have nilpotency class 2 . The other possibility is that the factor goup has nilpotency class 3. From the result in Section 2, groups like this can still only have nilpotency class four, but the structure of the factor group has a very specific form, as shown in Ormerod [6]. We consider groups like this on two generators, and note that they are metabelian, and since we restrict ourselves to $p>3$, the groups are regular. We state and prove the following theorem.

THEOREM 5.1. Let $r$ and $s$ be positive integers and let $G(r, s)$ be the group defined by the following relations:

$$
\begin{aligned}
G(r, s)=\langle a, b: & a^{p^{s+2 r}}=b^{p^{s+2 r}}=1, \\
& {[b, a, a, a]=a^{p^{s+r}},[b, a, b, b]=1, } \\
& {[b, a, a, b]=b^{p^{s+r}}\left[a^{p^{r}}, b\right]=\left[b^{p^{r}}, a\right]=[b, a, b, a], } \\
& {[b, a, a, a, b]=[b, a, a, b, a]=[b, a, a, b, b]=1\rangle }
\end{aligned}
$$

If $G$ is a 2-generator p-group of Wielandt length 3 and if $G / \omega(G)$ has nilpotency class 3 , then $G$ is a homomorphic image of $G(r, s)$ for some integers $r$ and $s$.

Proof: Let $G$ be a $p$-group of Wielandt length 3 with generators $a$ and $b$. Further assume that $G / \omega(G)$ has nilpotency class 3. From Ormerod [6] this means that $G / \omega(G)$ is a homorphic image of a group

$$
H(p, r)=\left\langle x, y:[y, x, x]=x^{p^{r}},[y, x, y]=y^{p^{r}}, x^{p^{2 r}}=y^{p^{2 r}}=1,[x, y]^{p^{r}}=1\right\rangle .
$$

From $2.1 G$ must have class 4, and we assume that the generators of $G$ are chosen in such a way that

$$
\omega(G) \supseteq\left\{a^{p^{2 r}}, b^{p^{2 r}},[b, a]^{p^{r}}, a^{-p^{r}}[b, a, a], b^{-p^{r}}[b, a, b]\right\}
$$

for some integer $r \geqslant 1$. Since elements of the Wielandt subgoup are second central, and since $a^{-p^{r}}[b, a, a]$ and $b^{-p^{r}}[b, a, b]$ are in the Wielandt subgroup, we can deduce that $[b, a]^{p^{r}}$ must be central in $G$. We also find that $a^{p^{2 r}}$ and $b^{p^{2 r}}$ must be central. For the possible non-central elements we put

$$
\left[a^{-p^{r}}[b, a, a], a\right]=a^{\mu},\left[a^{-p^{r}}[b, a, a], b\right]=b^{\mu},\left[b^{-p^{r}}[b, a, b], a\right]=a^{\nu},\left[b^{-p^{r}}[b, a, b], b\right]=b^{\nu}
$$

where $\mu$ and $\nu$ are integers. This simplifies to

$$
[b, a, a, a]=a^{\mu},[b, a, a, b]=b^{\mu}\left[a^{p^{r}}, b\right]=[b, a, b, a]=a^{\nu}\left[b^{p^{r}}, a\right],[b, a, b, b]=b^{\nu} .
$$


Write $\mu=m p^{s+r}$ and $\nu=n p^{t+r}$ where $(m, p)=(n, p)=1$ and $s, t \geqslant 1$. We shall assume that $s \leqslant t$. The condition that $G$ has nilpotency class 4 allows us to draw several conclusions:

1. $a^{p^{s+r}}, b^{p^{t+r}} \in \zeta(G)$,

2. weight three and weight four commutators have order at most $p^{r}$,

3. the order of $a$ is at most $p^{s+2 r}$,

4. $b^{p^{s+r}} \in \zeta(G)$, and

5. the order of $b$ is at most $p^{s+2 r}$.

This means that we can define a group $G$ with generators $a$ and $b$ and satisfying the following relations:

$$
\begin{aligned}
a^{p^{s+2 r}}=b^{p^{s+2 r}} & =1, \\
{[b, a, a, a] } & =a^{m p^{s+r}}, \quad[b, a, b, b]=b^{n p^{t+r}} \\
{[b, a, a, b] } & =b^{m p^{s+r}}\left[a^{p^{r}}, b\right]=a^{n p^{t+r}}\left[b^{p^{r}}, a\right]=[b, a, b, a]
\end{aligned}
$$

We now want to show that we can simplify this notation and we do this in two steps. In the first step assume that $t<s+r$ and put $x=a^{\alpha} b$ where $\alpha=\varepsilon p^{t-s}$ and $\varepsilon$ is chosen so that $n+2 m \varepsilon=0 \bmod p^{s+r-t}$. The value of $\alpha$ ensures that $\left(a^{p^{s+r}}\right)^{\alpha}=1$. This now means that the relations of $G$ can be written:

$$
\begin{aligned}
a^{p^{s+2 r}}=x^{p^{s+2 r}} & =1, \\
{[x, a, a, a] } & =a^{m p^{s+r}}, \quad[x, a, x, x]=1 \\
{[x, a, a, x] } & =x^{m p^{s+r}}\left[a^{p^{r}}, x\right]=\left[x^{p^{r}}, a\right]=[x, a, x, a] .
\end{aligned}
$$

This is exactly the situation we would get in the first set of relations for the cases $t \geqslant r+s$.

For the second step put $u=a^{\alpha}$ and $v=x^{m}$ where $\alpha$ is chosen so that $\alpha m=$ $1 \bmod p^{r}$. We now get the following presentation for $G$ :

$$
\begin{aligned}
u^{p^{s+2 r}}=v^{p^{s+2 r}} & =1, \\
{[v, u, u, u] } & =u^{p^{s+r}}, \quad[v, u, v, v]=1 \\
{[v, u, u, v] } & =v^{p^{s+r}}\left[u^{p^{r}}, v\right]=\left[v^{p^{r}}, u\right]=[v, u, v, u] .
\end{aligned}
$$

If additional relations are added to ensure that the group has class four, this is now in the form stated in the theorem.

We now show that any group with the presentation given in the theorem is in $\mathcal{W}_{3}$. To do this we show that

$$
\omega(G(r, s)) \supseteq\left\{a^{p^{2 r}}, b^{p^{2 r}},[b, a]^{p^{r}}, a^{-p^{r}}[b, a, a], b^{-p^{r}}[b, a, b]\right\} .
$$


It can be readily shown that all the elements listed, with the exception of $a^{-p^{r}}[b, a, a]$, are central in $G(r, \dot{s})$. So let $w=a^{-p^{r}}[b, a, a]$ and let $g$ be any element in $G(r, s)$. We can write $g=a^{\alpha} b^{\beta} u$ where $u \in \gamma_{2}(G(r, s))$ and $0 \leqslant \alpha, \beta \leqslant P^{r+s}$. The following calculation shows that $w \in \omega(G(r, s))$.

$$
\begin{aligned}
{[w, g] } & =\left[a^{-p^{r}}[b, a, a], a^{\alpha} b^{\beta} u\right] \\
& =\left[a^{-p^{r}}, a^{\alpha} b^{\beta}\right]\left[[b, a, b], a^{\alpha} b^{\beta}\right] \\
& =\left[a^{-p^{r}}, b\right]^{\beta}[b, a, a, b]^{\beta}[b, a, a, a]^{\alpha} \\
& =a^{\alpha p^{s+r}} b^{\beta p^{s+r}} \\
& =g^{p^{s+r}} .
\end{aligned}
$$

This confirms that $w$ is in $\omega(G(r, s))$ and concludes the proof of the theorem.

At this stage it is worthwhile to make a few comments about $G(r, s)$. We first assume that $s \geqslant r$. The following points enable us to determine the order of $G(r, s)$. For simplicity we just use the notation $G$.

1. $\gamma_{4}(G)=\langle[b, a, a, a],[b, a, b, a]\rangle$. Each of these commutators has order $p^{r}$, and we conclude that $\left|\gamma_{4}(G)\right|=p^{2 r}$.

2. $\gamma_{3}(G)=\langle[b, a, a],[b, a, b]\rangle$ modulo $\gamma_{4}(G)$. Again, each of these commutators has order $p^{r}$, implying that $\left|\gamma_{3}(G) / \gamma_{4}(G)\right|=p^{2 r}$.

3. $\gamma_{2}(G)=\langle[b, a]\rangle$ modulo $\gamma_{3}(G)$ and $|[b, a]|=p^{r}$ modulo $\gamma_{3}(G)$. Hence $\left|\gamma_{2}(G) / \gamma_{3}(G)\right|=p^{r}$.

4. $G=\langle a, b\rangle$ modulo $\gamma_{2}(G)$ and $|a|=|b|=p^{s+r}$ modulo $\gamma_{2}(G)$. Hence, $\left|G / \gamma_{2}(G)\right|=p^{2 s+2 r}$.

5. From this we conclude that $|G|=p^{7 r+2 s}$.

If $s<r$ then it is possible to show that $b^{p^{r+2 s}}=1$ and hence that $|G|=p^{6 r+3 s}$.

In the comments above it was noted that the last non trivial term of the lower central series has two generators, in contrast to the groups of the previous section. In looking at the question of the nilpotency class of groups of Wielandt length four, it is already known that these groups may have nilpotency class six. An example of such a group is provided in Ormerod [5, Section 3], and in this example the factor group $G / \omega(G)$ is one of the groups $G(r, s)$, with $r=s=1$. Whether this provides a more general indication as to when there may be a difference of two between the nilpotency class of a group and its factor group via the Wielandt subgroup is too early to tell.

Some 3-generator groups have been constructed, but these are all metabelian, and at present the general picture is unclear.

\section{REFERENCES}

[1] A. Ali, Supersoluble groups of Wielandt length two, Ph. D. Thesis (Australian Nationai 
University, Australia, 1997).

[2] C. Cooper, 'Power automorphisms of a group', Math. Z. 107 (1968), 335-356.

[3] C. Hobby, 'Finite groups with normal normalizer', Canad. J. Math. 20 (1968), 1256-1260.

[4] S.K. Mahdavi, 'A classification of 2-generator 2-groups with many subgroups 2-subnormal', Comm. Algebra 15 (1987), 713-750.

[5] E.A. Ormerod, 'On the Wielandt length of metabelian p-groups', Arch. Math. 57 (1991), 212-215.

[6] E.A. Ormerod, 'Groups of Wielandt length two', Math. Proc. Cambridge Phil. Soc. 110 (1991), 229-244.

[7] E. Schenkman, 'On the norm of a group', Illinois J. Math. 4 (1960), 150-152.

[8] H. Wielandt, 'Über den Normalisator der subnormalen Untergruppen.', Math. Z. 69 (1958), 463-465.

Mathematics Department

School of Mathematical Sciences

The Australian National University

Canberra ACT 0200

Australia 